\title{
Variations
}

Variations

Revue internationale de théorie critique

$21 \mid 2018$

L'industrie de la culture : version originale

\section{Une social-démocratisation de la critique sociale? De la réification chez Axel Honneth}

Antoine Printz

\section{(2) OpenEdition}

Journals

Édition électronique

URL : http://journals.openedition.org/variations/933

DOI : 10.4000/variations.933

ISSN : 1968-3960

Éditeur

Les amis de Variations

Référence électronique

Antoine Printz, « Une social-démocratisation de la critique sociale ? De la réification chez Axel Honneth », Variations [En ligne], 21 | 2018, mis en ligne le 05 avril 2018, consulté le 21 avril 2019. URL : http:// journals.openedition.org/variations/933; DOI : 10.4000/variations.933

Ce document a été généré automatiquement le 21 avril 2019

Les ami•e•s de Variations 


\title{
Une social-démocratisation de la critique sociale? De la réification chez Axel Honneth
}

\author{
Antoine Printz
}

1 Le reproche en anachronisme est connu et éculé : la théorie marxiste serait impropre à penser une nouvelle réalité, parfaitement différente de celle de son contexte sociohistorique d'émergence. Il importerait donc d'actualiser ce cadre théorique afin de le rendre congruent avec l'ordre social contemporain, profondément différent de la réalité prolétarienne de l'entre-deux-siècles précédent. Axel Honneth par exemple s'est attelé à cette tâche, fort de la légitimité d'un héritage francfortois qu'il prétend pourtant dépasser. À cette fin, il s'efforde depuis dix ans, à actualiser un concept (presque) centenaire du marxisme occidental, celui de réification. Il nous semble que le travail qui en résulte n'a malheureusement pas été à la hauteur de la volonté de renouveler la première Théorie critique, d'inspiration marxiste.

2 Prenant cette entreprise comme relativement caractéristique, nous montrerons comment l'adaptation (forcée, voire forcenée) du thème lukácsien à des appareils théoriques qui lui sont complétement étrangers, censée permettre la résolution de ses apories et constituer un adjuvant à une réception nouvelle de la théorie, résulte en une perte critique notable. Le propos n'est en aucun cas celui d'un dogmatisme fermé à l'idée d'une cumulativité dans les sciences sociales: il est bien entendu que l'apport de pensées neuves à un appareil conceptuel aussi daté que l'est le logiciel marxiste est digne d'intérêt. Cependant, dans ce cas précis, qui est peut-être symptomatique, cette relecture semble résulter en la perte complète de ce qui faisait la radicalité de Lukács, ses outrances et son ampleur exclusive certes, mais aussi, et c'est sans doute profondément lié, son véritable potentiel critique. Nous pourrons voir, après présentation des arguments en faveur du dépassement théorique du marxisme occidental, comment cette reprise honnethienne de Lukács consiste en fait en une perte de sa radicalité critique. En voulant rendre pertinent le thème de la réification, au nom d'une prétendue nécessité de dépassement de la pensée 
marxiste, Honneth ne l'aurait-il pas finalement rendu adapté ? Et ce au risque de situer la théorie critique dans l'horizon réformiste.

\section{Le marxisme occidental impropre à penser le monde ?}

Dans un article annexe à son ouvrage de sociologie générale, Danilo Martuccelli identifie de manière assez pertinente ce qui constitue, pour lui, les deux problèmes centraux empêchant l'opérationnalité sociologico-critique de la théorie marxiste: sa subordination révolutionnaire et sa réduction de la praxis à la seule activité productive dans son sens restreint ${ }^{1}$. Qu'est-ce à dire ? Premièrement, en thématisant sa critique de la modernité capitaliste sous la forme finale - et finaliste - d'une eschatologie révolutionnaire, le marxisme, et le sociologue vise tout particulièrement sa filiation lukácsienne, se priverait de la possibilité d'une analyse critique réellement efficace. Les concepts tels que ceux de réification ou d'aliénation peineraient à constituer de bons opérateurs sociologiques dès lors qu'ils sont serviteurs d'un projet qui les dépasse, à partir duquel la critique se passe. Agents de cette prospective de la révolution prolétarienne, les théoriciens marxistes seraient alors, bien que souvent brillants, peu lisibles en-dehors de cette projection théorique, et dès lors difficilement intégrables à une pensée qui ne s'identifierait pas à la cause prolétarienne, ou qui même prétendrait, au nom d'une fameuse neutralité attribuée à Max Weber, séparer les registres du théorique et de l'axiologique. Deuxièmement, l' herméneutique du travail dans laquelle s'est enfermée cette tradition de pensée lui empêche une sortie de l'ancrage productiviste dommageable tant du point de vue théorique en ce qu'il constitue une réduction du champ d'analyse de la praxis humaine, que d'un point de vue politique tant on sait les (remises en) questions posées par la centralité du travail dans nos sociétés. À l'heure du chômage massif stabilisé et d'une baisse tendancielle $\mathrm{du}$ travail social nécessaire, induite par la hausse des gains de productivité et un support technologique à la production sans cesse croissant, faire du travail salarié la base de l'analyse critique des pathologies sociales relèverait au mieux d'une nostalgie théorique non pertinente, au pire d'une inconséquence empirique condamnable.

4 La théorie lukácsienne de la réification, point nodal du " marxisme occidental ${ }^{2}$ ", seraitelle alors à jeter aux oubliettes d'une pensée trop encombrée par ses références d'un autre temps? Seule trace romantique d'un passé glorieux de la critique européenne, dans une forme des plus étincelantes, mais d'une inutilité proportionnelle au génie de son propos? L'impasse opérationnelle à laquelle se confronte la pensée marxiste semble plus ou moins acquise à la plupart de ses commentateurs; et pourtant, on lui remarque, malgré cela, une postérité encore vivace, voire même renaissante. Ainsi, une occurrence nouvelle du thème de la réification dans la littérature montre à l'inverse du fatalisme mortifère un certain retour en force de ces catégories, si tant est qu'elles n'aient jamais totalement disparues.

5 Axel Honneth participe à cette (re)prise d'importance du concept dans le débat critique en publiant en 2007 un ouvrage simplement titré La Réification ${ }^{3}$. Ce travail s'inscrit explicitement dans le projet d'une réactualisation sociologique et critique de cet opérateur conceptuel. Cette entreprise semble pourtant devoir payer le prix fort : celui de l'arrachement quasi-total au terreau marxiste. Il s'agit alors de la reprise partielle et partiale d'un héritage dont le légateur principal aurait sans aucun doute refusé les formes et contenus nouveaux. Qu'en est-il alors d'un legs tant dévié dans son essence même ? La 
nécessité, à laquelle a, au premier abord, souscrit Honneth, de retirer tout ancrage marxiste à la pensée marxiste pour la rendre adéquate à penser le social et ses pathologies doit être questionnée en ce sens exact.

\section{Lukács selon Lukács}

6 Le phénomène de la réification a été théorisé par György Lukács, philosophe hongrois, au début du XXème siècle dans le livre Histoire et conscience de classe, et plus particulièrement dans son article central: La réification et la conscience du prolétariat ${ }^{4}$. Le génie de Lukács réside dans sa capacité de synthèse, dans une conceptualisation cohérente et conséquente, entre ce qui constitue sans doute les deux plus féconds héritages critiques allemands. Si Lukács est bien sûr un marxiste, il est ce que Löwy a, à juste titre, nommé un marxiste romantique ${ }^{5}$. Ses référents théoriques sont en effet tant à trouver du côté de Marx que de la tradition allemande de la Kulturkritik; c'est ce qui fait la profondeur de ses développements, c'est aussi ce qui fera la postérité insolite de ce «livre maudit du marxisme ${ }^{6} \%$.

7 Ambition grandiose que de pointer le phénomène pathologique de la modernité capitaliste, qui souffre les défauts de ses qualités mais reste néanmoins absolument géniale dans son entreprise : résolution et dépassement critique du rapport au monde de l'homme du capitalisme moderne, essentiellement tronqué, dont les parents sont la marchandisation généralisée et la rationalisation désenchantée. Il n'est pas le premier, bien sûr, à mettre en lumière l'avènement dans la société occidentale de cette nouvelle manière pour l'homme d'habiter son monde en ce début de siècle : la tradition critique le précède, que cela soit au sein de la pensée marxiste ou dans la tradition philosophique de l' irrationalisme allemand. Cependant, il est le premier à joindre ces deux traditions d'apparence antagonistes, donnant lieu là, par « la tentative la plus audacieuse et la plus fertile de repenser la constitution du marxisme ${ }^{7}$ ", à un travail permettant de penser les effets structurels du capitalisme sur l'homme et son monde, comme phénomène amont et nécessaire à celui qui occupe l'analyse marxiste classique orthodoxe, celui de l'exploitation.

8 La conceptualisation lukácsienne se base sur l'intuition marxiste du caractère fétiche de la marchandise, décrivant le fait que la relation entre les hommes est occultée par celle entre marchandises, couplée à l'analyse wébérienne de la rationalisation : la réification est le processus par lequel un être, une forme de vie, une relation qui n'est pas une chose devient néanmoins (perçue $\left.{ }^{8}\right)$ comme telle. La réification des formes sociales naît de ce phénomène proprement capitaliste qu'est l'universalisation de la forme marchande, qui traduit « l'ensemble des manifestations vitales de la société ${ }^{9}$ ». Cette universalisation comme prise d'importance jusqu'alors inconnue de la forme marchande est bien plus qualitative que quantitative. Bien sûr elle se manifeste par une augmentation numérique des objets devenus marchands, mais cette prise d'importance quantitative n'est que le fait de la transformation qualitative de la société marchande, au sein de laquelle la marchandise n'est plus contingente mais nécessaire et totale : l'organisation productive de la société capitaliste vise la production de valeurs d'échange, non plus de valeur d'usage. Le produit humain acquière la forme de marchandise, autonome et inhumaine. Et cette forme de devenir peu à peu la " forme universelle qui façonne la société ${ }^{10}$ ", aboutissant à la domination marchande et l'extériorisation objectale du monde social. 
C'est l'organisation sociale capitaliste moderne qui induit la prévalence qualitative des " relations marchandes [...] subjectives et objectives ${ }^{11}$ » et dont découle le phénomène réificatoire. Cette étiologie du phénomène est essentielle à identifier parce qu'elle constitue l'un des points de divergence majeurs dans la reprise honnethienne, et qu'elle a une implication centrale pour la critique. Quelle est donc précisément la source de la réification des rapports sociaux? Qu'est-ce qui induit ce rapport particulier de l'homme à son monde? Quelle instance socialisatrice génère l'homme spectateur à la conscience réifiée ? C'est selon Lukács l'organisation capitaliste du travail basée sur la division rationnelle du processus de production.

\section{L'organisation capitaliste du travail et la marchandisation du travailleur}

10 La modernité a vu l'avènement de processus de production rompant avec l'unicité organique qui les caractérisait dans les sociétés traditionnelles. L'abstraction et la division du travail ont généré des modalités de production nouvelles, caractérisées par l'élimination progressive de toute dimension qualitative et humaine au profit de la froide rationalité spécialisée, basée sur le calcul et la prévisibilité. Cette rationalisation rentabilisatrice des processus de production provoque pour l'homme la perte de la totalité puisque son mode d'action pratique est proprement décomposé et analytique. Le monde n'est alors plus appréhendé que dans sa facticité, perdant son unité organique et intégrée pour la personne du travailleur : celui-ci est incorporé au processus de travail en tant qu'il est un rouage qui vient dans un flux déjà-là, dont il ne peut saisir la totalité et au sein duquel il est interchangeable - un homme d'une heure vaut un autre homme d'une heure disait Marx.

La division du travail provoque l'advenir d'un « travail rationalisé, parcellisé, standardisé ${ }^{12}$ ", et dès lors d'un ouvrier - et donc d'un homme puisque, et ce au plus grand désespoir du capital, l'un ne peut être sans l'autre - adapté, c'est-à-dire parcellaire, spécialisé et désindividualisé ${ }^{13}$ dans la standardisation forcée de la force de travail. Le travailleur est dorénavant inséré comme " partie mécanisée dans un système mécanique qu'il trouve devant lui, achevé et fonctionnant dans une totale indépendance par rapport à lui ${ }^{14}$ ».L'abstraction du travail, qui fonde l'égalité formelle entre les choses et donc la marchandise, fait des sujets humains les seuls porteurs d'une quantité de travail abstrait, niant par-là l'importance de l'individu dans sa dimension expressive, et le travail dans sa dimension expérientielle.

La réification du travail ne se limite cependant pas au travail dans l'usine dont elle est un cas franc, le travail intellectuel se trouve également réifié dans la société capitaliste. Le journaliste constitue en ce sens la figure paradigmatique de l'aliénation marchande. La structuration marchande du travail du journaliste le contraint à voir et user objectivement de ses traits les plus intimes et qualitatifs, personnels : «la subjectivité elle-même, le savoir, le tempérament, la faculté d'expression, deviennent un mécanisme abstrait ${ }^{15}$ ». Le journaliste voit son activité lui être aliénée au même titre que l'ouvrier d'usine, sauf que dans son cas, sa force de travail, ce sont son opinion, sa plume et son expression, qui se trouvent réduites à une expression marchande. En ce sens, l'expérience journalistique est le «point culminant de la réification capitaliste ${ }^{16}$ ». Les processus du travail journalistique subissent la même expropriation que la force de travail ouvrier dans l'usine, devant répondre aux finalités qui ne sont pas les siennes et qui imposent 
un morcellement de l'individu. Le journaliste est porteur d'une marchandise, sa plume, qu'il vend à sa rédaction, comme l'ouvrier vendait sa force de travail physique à l'entreprise ; tous deux se trouve divisés intérieurement dès lors qu'ils mettent une partie d'eux dans des processus de production qui les dépassent et leur astreignent la tâche spécifique et partielle qu'ils ont à produire, et on retrouve là l'esprit du manifeste :

[C]es travailleurs sont obligés de se vendre morceau par morceau tels une marchandise ; et, comme tout autre article de commerce, ils sont livrés à toutes les vicissitudes de la concurrence, à toutes les fluctuations du marché. ${ }^{17}$

\section{Extension à l'ensemble de l'organisation sociale}

Selon Lukács, le phénomène de la réification ne se limite pas à ce type spécifique d'aliénation du travailleur. La conscience réifiée applique ses structures exhaustivement au monde humain organisé. Les relations propres au mode de production, qui faisaient de l'homme un contemplateur de sa propre activité productive, se généralisent à l'ensemble des manifestations vitales de sorte que « la personnalité devient le spectateur impuissant de tout ce qui arrive à sa propre existence, parcelle isolée et intégrée à un système étranger ${ }^{18}$ ». L'adaptation humaine requise dans la sphère productive s'étend dans ses modalités particulières à l'ensemble de l'existence sociale de l'homme. La société capitaliste se structure selon le modèle de l'usine et «le destin de l'ouvrier devient le destin de toute la société ${ }^{19}{ }^{\prime}$.

Il y a dès lors amplification de la domination inhérente à l'organisation du travail capitaliste " au-delà de son terrain d'origine ${ }^{20}{ }^{\prime}$. C'est là la force d'Histoire et conscience de classe, d'avoir fait sortir l'analyse du capitalisme moderne des conditions de l'usine pour montrer, dans une inspiration tragique, comment la réification impacte l'ensemble des manifestations sociales.

Les plus réfractaires des commentateurs noterons bien sûr que toutes les sociétés humaines connaissent ce type d'objectivation, de routinisation pratique. Il semble en effet qu'il soit le lot de toute organisation sociale humaine de réduire la diversité d'action au sein de procédures et d'institutions plus ou moins formalisées, qui permettent une vie sociale stabilisée. Cependant la forme capitaliste a, selon Lukács, une spécificité qui est celle de l'autonomisation des institutions sociales, et de leur retournement contre la vie réelle. L'institution capitaliste moderne n'est alors plus au service de l'homme dans la stabilisation de son existence sociale, mais lui aliéne l'authenticité de son activité vitale par son asservissement à la rationalité marchande. La réification décrit ce processus, particulier à l'organisation capitaliste, de l'objectivation des formes sociales ne servant pas les finalités humaines mais, dans un geste tout tautologique, le maintien dans l'existence, sans cesse renforcé, de ces formes mêmes et au sein desquelles, comme le disait Lukács dans des entretiens donnés vers la fin de sa vie, le fait individuel semble égaler zéro ${ }^{21}$.

Il semble donc qu'on assiste à la dépossession de l'individu de son action existentielle, l'incapacitant dans son action véritable, c'est-à-dire qui ne soit pas vaine dans le monde extérieur. Stieg Dagerman exprimait, dans son testament philosophique, la même intuition de l'oppression de l'homme par le monde qu'il a créé, montrant les liens, bien que décriés par le Lukács tardif, entre ce marxisme et l'existentialisme: « ce que je serai alors contraint de reconnaitre, c'est que l'homme a donné à sa vie des formes qui, au moins en apparence, sont plus fortes que lui ${ }^{22}$ ». Le monde, humain pourtant, empêche le triomphe 
de la liberté concrète de l'homme «non pas hors du monde et des choses et des produits humains ${ }^{23}$ » mais à l'inverse, précisément dans et sur le monde humain.

\section{La reprise honnethienne}

18 C'est sur ces bases théoriques qu'Axel Honneth va tenter une intégration des développements lukácsiens à sa propre matrice de pensée - celle de la reconnaissance ${ }^{24}$-, afin de leur (re)donner une opérationnalité théorique et critique. Le Francfortois considère devoir débarrasser le concept d'une série de ses traits marquants pour pouvoir le réintégrer au paradigme de la reconnaissance, réduisant le concept à un processus dispositionnel de déni de reconnaissance, s'épargnant la réflexion socio-historique quant aux causes d'un tel état de fait.

19 La parution de son ouvrage La Réification, consacre cette volonté d'une ré-exploration de la catégorie marxiste au regard de la théorie de la reconnaissance, dont l'ancrage pragmatiste pose les bases d'une empiricisation de la construction d'une grammaire morale, dans la confrontation concrète à la réalité et au sentiment d'injustice qu'elle peut générer. Honneth traduit et transpose les idées directrices du fameux texte lukácsien selon cette volonté marquée de fonder la théorie critique dans le sentiment moral des individus, considérant que le grand manquement de la théorie lukácsienne réside dans son déficit normatif. La question est dès lors posée en termes moraux. Honneth s'oppose en effet à l'entreprise lukácsienne de construire le concept sans se référer aucunement à la question morale.

Ce n'est pas parce qu'un certain comportement, le comportement 'réifiant', viole les présupposés ontologiques de notre action quotidienne qu'il passe pour problématique ou pour faux, mais parce qu'il viole certains principes moraux. ${ }^{25}$

21 Lukács posait en effet la problématique en termes d'ontologie - réification comme dévoiement par rapport à la vérité de l'être social -, Honneth entend fonder une théorie sociale à teneur normative, et ce en accord avec son projet théorique et critique basal. Il est donc une différence préjudicielle centrale entre les deux auteurs sur ce point. D'un côté, Lukács analysait la réification comme une pratique dévoyée par rapport à la pratique humaine originaire, c'est-à-dire la praxis authentique fondée sur une anthropologie sociale marxienne, voire une ontologie sociale - le monde est production humaine. De l'autre, Honneth voit la réification comme comportement acquis socialement, comme une disposition, qui contrevient à l'attitude morale de reconnaissance, caractéristique d'un sujet humain « de part en part moral, qui participe avec toute son affectivité à la vie de la société et y réagit de manière normative ${ }^{26}$ 》.

22 Le Francfortois procède donc à l'amoralisation des pratiques réifiantes. Ce n'est selon lui qu'à ce prix qu'elles pourront être critiquées comme pathologie sociale. En posant ses développements sous un tel patronage, il se trouve nécessairement éloigné de son prédécesseur Hongrois qui voyait la réification comme un " ensemble d'habitudes et d'attitudes qui contredisent les règles de ce que serait une forme plus originaire ou encore meilleure de pratique humaine ${ }^{27}$ ». 


\section{Contre la réduction marxiste}

Cette pensée lukácsienne, par trop idéaliste, ne convient pas au philosophe francfortois et l'amène à faire la proposition d'une relecture basée sur «l'analyse de la teneur normative de la vie sociale " par le biais d'une " herméneutique de la vie sociale, sur la base d'une phénoménologie des expériences de l'injustice ${ }^{28}$ ». Le geste n'est pas véritablement neuf, il correspond à la volonté fondatrice d'Horkheimer et Adorno d'une théorie critique appuyée sur une heuristique du malaise, c'est-à-dire sur l'identification des mutilations de la vie sociale ${ }^{29}$. Ce n'est cependant pas là que la rupture est la plus nette, mais bien plutôt dans les présupposés déductifs sur lesquels se posent le diagnostic pathologique.

24 En effet, selon Honneth, le point de vue marxiste " réduit [...] les relations de reconnaissance entre les êtres humains à la seule dimension de la satisfaction matérielle ${ }^{30}$ ». Le marxisme classique, et Lukács, situe nécessairement l'intérêt préscientifique pour l'émancipation dans la classe prolétarienne. Ce que Honneth refuse, dès lors qu'il ne situe plus la question des pathologies du social sur le seul pan de l'abstraction de l'activité humaine dans les processus de production capitaliste, ni même d'ailleurs dans l'injustice de l'inégalité de la participation économique.

$C^{\prime}$ est pour d'excellentes raisons que nous avons perdu l'idée selon laquelle on pourrait attribuer des intérêts émancipatoires ou des expériences à un groupe de personnes dont le seul point commun est la situation socio-économique. ${ }^{31}$ Avec l'abandon de la prédominance des facteurs socio-économiques infrastructurels, disparaît quasi automatiquement la classe sociale. Ce dont Honneth ne se cache d'ailleurs pas, jugeant inadéquate une analyse en seuls termes de classes, et qui conférerait en outre à la classe prolétarienne le rôle messianique d'unique adjuvant de la révolution. La philosophie moderne ne peut donc plus restreindre le travail de catégorisation sociologique à la seule perspective socio-économique.

Il apparaît qu'une théorie des classes ajustée à la société capitaliste ne peut se borner à l'inégale répartition des biens matériels, mais doit aussi prendre en compte la répartition asymétrique des chances sur le plan culturel et psychique. Je vise par-là l'inégalité difficilement mesurable, mais parfaitement attestée, entre les différentes classes dans l'accès à la culture, à la reconnaissance et à un travail personnalisé. ${ }^{32}$

\section{Que reste-t-il alors à critiquer?}

Qu'est-ce que la réification sur de telles bases alors? Beaucoup de choses sont perdues dans la reprise mais la réification comme prééminence du rapport cognitif au monde est développée et solidifiée par Honneth. Point de conscience prolétarienne révolutionnaire bien sûr, mais la perte du rapport d'affectivité, ou récognitif, de l'homme à ce qui l'entoure. La connaissance devient la manière première d'être au monde, ou plutôt d'observer le monde puisque ce scientisme quotidien empêche tout réel engagement. L'attitude scientiste à l'égard de la réalité consiste en l'évincement, ou le remplacement, des relations de reconnaissance.

J'aimerais d'une certaine façon comprendre le scientisme et ses équivalents dans les conceptions sociales, impliquant des relations instrumentales avec autrui, comme le pendant négatif de l'infrastructure de la reconnaissance sociale [...]. ${ }^{33}$ 
29 La connaissance scientiste court-circuite l'étape primordiale récognitive, celle de «[1'] affirmation de l'autre ${ }^{34} »$. L'ensemble de la construction honnethienne dans sa reprise du thème de la réification est basée sur " la thèse selon laquelle l'attitude participante et engagée précède la saisie neutre de la réalité ${ }^{35}$ »; et que les pratiques réifiantes consiste en un oubli de la reconnaissance, en «l'adoption par les sujets [...] d'une posture de simple connaissance et non de reconnaissance ${ }^{36} \%$. Honneth calque donc sa reprise de la réification sur sa théorie de la reconnaissance, l'insérant dans la dichotomie - non oppositive - connaissance/reconnaissance. La réification, c'est le primat de l'attitude de connaissance, sans le préalable de la reconnaissance ${ }^{37}$.

\section{La sortie de la focalisation capitaliste marchande}

Il reste une dernière question à élucider pour comprendre parfaitement la reprise de Honneth: comment la reconnaissance - comme lien affectif et engagé au monde originaire - peut-elle tomber dans l'oubli ? Pour Lukács, nous l'avons vu, c'était la généralisation de la forme marchande et le processus de rationalisation qui étaient à l'origine de la réification comme expropriation de l'homme du monde social humain. Cette théorie n'est pas recevable pour Honneth, il lui faut cependant expliquer comment la reconnaissance peut être oubliée dans la pratique : quels sont les ressorts causaux de cette inconscientisation chez l'homme de son lien primordial affectif - et préréflexif - au monde ? Le rejet de la vue lukácsienne sur la généralisation totalisante de la sphère marchande mène Honneth à devoir proposer au phénomène réificatoire une causalisation alternative. C'est ce qu'il fait dans la dernière partie de son essai - Les sources sociales de la réification. L'étiologie qu'il y avance est cependant relativement obscure et imprécise. Il semble qu'Honneth ne parvienne pas à proposer une démonstration claire des facteurs explicatifs de la réification dès lors qu'il a prétendu sortir le concept de son ancrage causal capitaliste ${ }^{38}$.

31 Honneth dégage en vérité deux facteurs desquels sont issues, selon lui, les pratiques intersubjectives réifiantes : (1) lorsque le rapport au monde et aux autres est médié par des préjugés et stéréotypes ancrés et, (2) lorsque le but de l'activité ou de la posture de connaissance s'autonomise.

[O]u bien [les individus] participent à une pratique sociale dans laquelle la simple observation d'autrui devient une fin en elle-même, à tel point que tout conscience d'une relation préalable est effacée [(2)], ou bien ils se laissent conduire dans leurs activités par un système de convictions qui les contraint à dénier cette reconnaissance originelle [(1)]. 39

La première source de la réification est selon lui due à l'habitude de catégorisation typifiante, d'un «système de convictions réifiant ${ }^{40}$ ». Le travail d'ordonnancement et de tri catégoriel du monde social découle en l'abstraction des qualités humaines particulières, lorsque des «systèmes de convictions conduisent sans ambiguïté à la définition de types réifiés de groupes de personnes ${ }^{41}$ ». Sous l'emprise de raccourcis cognitifs, l'individu appréhende l'autre indépendamment de sa valeur et de ses qualités personnelles - comme un exemplaire plutôt que comme un exemple ${ }^{42}$. Honneth note qu'il ne s'agit pas d'une attitude cognitive neutre mais d'un réel travail de hiérarchisation sociale et de distinction, il ne s'agit donc pas d'une opération intellectuelle mais de la construction de «stéréotypes commandés en général par des motivations hostiles dans un contexte de distinction sociale conflictuelle ${ }^{43}$ ». 

interprétation très stricte, couplé à l'arrachement à la causalité économique, restreint l'analyse aux seuls cas extrêmes : «C'est seulement dans le cas de l'esclavage économique qu'on aurait affaire à des conceptions et des pratiques réifiantes ${ }^{47}$ ». Le champ critique, alors même que Honneth prétendait élargir et assouplir le concept, se trouve là terriblement restreint. Le concept semble en effet peu extensif, et s'il parvient à ramener à l'analyse des phénomènes omis par Lukács, il oublie tous les autres, qui étaient initialement couverts. Cette littérarité honnethienne provient peut-être d'une certaine mécompréhension de la formule consacrée où la réification est définie comme le phénomène durant lequel la relation entre personnes prend le caractère d'une chose. C'est en effet, et le point est primordial, d'abord la relation entre personnes, et non les personnes, qui apparaissent à l'homme comme des choses. conceptualisation de la réification, c'est sa littéralité : les cas de traitement réel en tant que choses sont limités à la galerie des horreurs humaines de l'esclavage aux génocides, c'est-à-dire qu'ils sont relativement circonscrits localement et temporellement. Ponctuations barbares de l'histoire, ce ne sont ni des attitudes générales, ni quotidiennes ; ce qui fondait pourtant le diagnostic lukácsien et a continué d'habiter sa descendance, que ce soit dans la Critique de la vie quotidienne de Lefebvre ou les écrits situationnistes. Il est donc là un certain échec honnethien à rendre compte d'une potentialité critique 
véritable et large, d'autant plus paradoxal qu'il prétendait réinscrire la réification dans les conditions empiriques contemporaines et en assouplir la définition.

\section{Conclusion}

Qu'en est-il alors de cette actualisation de la critique? Si ce mouvement de reprise peut être vu comme empli de bon sens, il n'en reste pas moins que quelque chose est perdu. Bien sûr, il peut paraître théoriquement sain d'arracher Lukács à Lukács, à son totalitarisme conceptuel et ses généralisations abusives. Il peut en être de même à l'égard de son héroïsation fantasmée de la classe prolétarienne. Cependant, le lecteur ne peut sortir de la lecture comparée des deux réifications qu'avec un certain goût de trop peu.

Lukács surprend par son faste, ses effets et le génie de ses développements. Honneth est certes peut-être plus réaliste, certes peut-être plus mesuré mais la démesure lukácsienne, son romantisme à la fois noir et optimiste, et son analyse pénétrante procurait un outil difficile en même temps que génial et infini. La traduction honnethienne semble paradoxalement déboucher sur une réduction de la perspective : l'intersubjectivisme radical des thèses donne lieu à l'impossibilité de voir la réification dans ses dimensions institutionnelles ou systémiques (étiologie) et politiques (épilogue). Et cela paradoxalement dès lors qu'Honneth prétendait sortir la réification de l'ornière réductionniste dans laquelle le concept était censé s'être enlisé.

En ce sens, de nombreux commentateurs ont reproché à Honneth sa reprise, notamment parce que celle-ci, en prétendant délivrer la réification de son bagage lukácsien encombrant a simplement rompu avec la possibilité véritable d'une critique. Angella pointe le risque qu'en voulant délivrer la théorisation lukácsienne de son inopérationnalité par excès, Honneth ne lui substitue une théorie inopérationnelle par minimalisme ${ }^{48}$. Le travail honnethien est ainsi qualifié durement par certains de ses lecteurs : échec pour Jütten ${ }^{49}$, dialyse conceptuelle ou lyophilisation du concept pour Charbonnier ${ }^{50}$, ou encore révisionnisme pour Morgan ${ }^{51}$.

Honneth propose une version fondée sur une anthropologie fondamentale déconnectée de toute donnée sociale ou historique, la réification n'est pas réellement expliquée. À tout le moins, elle est expliquée par une série de causalités secondes mais point de cause sociale première, qui expliquerait l'ensemble des causes secondes. On croit éventuellement deviner une théorie sociale d'inspiration bourdieusienne, qui placerait dans la compétition symbolique et la distinction sociale le principe premier de l'ensemble de certaines habitudes réificatoires. Il n'en reste pas moins que l'explication honnethienne est plus d'ordre mécanique que causale ou étiologique. Et la restriction intersubjective lui fait perdre tout rapport avec les réalités historiques.

Or, s'il est bien une perte centrale à laquelle Lukács n'aurait jamais consenti, c'est celle du caractère historique du concept de réification. En attachant causalement celui-ci à une constellation, relativement indéfinie, de pratiques sociales non liées spécifiquement au mode de production historique qu'est le capitalisme, Honneth arrache le phénomène réificatoire à son étiologie marchande et en fait une pathologie sociale humaine bien plus qu'une pathologie explicitement attachée à la modernité capitaliste. Nous trouverons alors la dernière critique de Honneth chez Lukács, qui dans La réification et la conscience du prolétariat adresse un passage à la science bourgeoise particulièrement adapté, comme par anticipation, au Francfortois. 

cependant, bien qu'ils voient les " conséquences humaines désastreuses » de ces phénomènes, ils ne parviennent à sortir de son immédiateté, ne les raccrochant pas à " leur terrain naturel capitaliste ${ }^{52}$ \%. Ces intellectuels en font alors des phénomènes éternels et vidés de toute origine capitaliste, opérant une naturalisation acritique de ce qu'ils entendaient dénoncer. C'est typiquement le cas de Simmel selon Lukács qui en reste « aux formes extérieures d'apparition de la réification ${ }^{53}$ \%.

Le philosophe hongrois, dans ces pages, souligne la difficulté de saisir l'origine infrastructurelle de ces phénomènes puisqu'ils englobent nécessairement l'ensemble des fonctions vitales de la société humaine, ne se limitant pas à la seule organisation de l'appareil productif. Cependant, en ne décelant par le terrain d'origine du phénomène réificatoire, ce type de science critique de la société porte le risque de son intégration. En se faisant critique des pathologies sociales sans pointer le capitalisme, elle s'en fait agent de légitimation. Là est toute la force de la science bourgeoise comme discours de légitimation du monde tel qu'il est.

47 C'est le matérialisme historique qui pourra réinscrire cette soi-disant nécessité nomologique au sein de la contingence historique et sociale qui l'a vu naître ; et permettra la sortie du blocage historique. De son point de vue, la bourgeoisie ne peut connaître, encore moins agir, la société réifiée puisqu'il y a une « intime interaction entre la méthode scientifique qui naît de l'être social d'une classe, de ses nécessités [...] et de l'être même de la classe ${ }^{54} \%$. La pensée bourgeoise est donc limitée intrinsèquement quant à sa possibilité d'accès à une vraie conscience.

48 N'est-ce pas le cas de la théorie honnethienne lorsqu'elle prétend faire sortir le phénomène de la réification de sa matrice théorique marxiste ? Faire de Honneth un intellectuel bourgeois est peut-être abusif. Il n'en reste pas moins que l'on peut trouver quelques affinités entre la science bourgeoise définie par Lukács et les développements honnethiens. Notamment, et c'est central, le fait de ne plus opérer de critique historique de l'économie politique qui génère une naturalisation du fonctionnement du monde tel qu'il est historiquement. Honneth ne parvient pas à penser le monde dans sa contingence historique, et à penser dès lors le progrès social en dehors de la réforme. Il opère en cela une sociale-démocratisation de la critique, qui s'accommode finalement de réalités que Lukács prétendait renverser.

49 D'aucuns jugeront cette refonte salvatrice, conférant enfin à la catégorie une pertinence sociologique opérationnelle. Cependant, en sortant des catégories centenaires qui fondaient la théorisation lukácsienne, nous pourrions dire qu'in fine, ce qui manque à la théorie de Honneth, c'est justement un peu d'impertinence. Qu'est-ce à dire? Il nous semble que la théorie honnethienne ne peut être vue que comme pertinente et peut rarement être réellement prise en défaut. C'est justement là que le bât blesse : elle non plus ne prend jamais réellement en défaut. La critique des génocides ou de la traite des êtres humains comme attitude amorale est fondée et trouvera partout soutiens. Il n'en reste pas moins que si le geste critique se résume à la dénonciation des massacres, de l'esclavage et de la marchandisation du corps, il est impropre à fonder les conditions théoriques d'une identification de l'ensemble des pathologies sociales et partant d'un réel revirement social.

50 Si la théorie est toujours pertinente, elle n'en manque pas moins de la vraie impertinence qui fonderait son potentiel critique véritable. La pertinence, c'est être adéquat mais aussi 
être adapté : certes la théorie de la réification par Honneth est absolument adéquate mais n'est-elle pas aussi malheureusement adaptée, ou à tout le moins adaptable? La théorie lukácsienne était évidemment démesurée, gigantesque et peu praticable mais elle était dans le même temps, et par-là même, foncièrement critique. En voulant la rendre directement praticable, sans excès et débordement, Honneth l'a tout simplement vidée de son contenu et en a fait une théorie minimale, à même de ne critiquer que le notoirement critiquable.

Le risque serait que Honneth ait produit une critique parfaitement récupérable. Ce n'était pas le cas de la théorie lukácsienne. Bien sûr, celle-ci était atrocement colossale et inadaptée. Elle était en outre difficile à user dans une perspective critique, a fortiori sociologique. Un reproche ne peut cependant lui être fait: celui d'être intégrable ou récupérable. Les écrits du jeune Lukács sont profondément et essentiellement critiques. L'eschatologie révolutionnaire lukácsienne souffre d'une thématisation trop dogmatique et d'une prétention exhaustive qui la perd, il n'en reste pas moins qu'elle modalise une finalité inadaptable, un autre ailleurs. Le risque de la théorie honnethienne n'est-il pas, à l'inverse, celui de son intégration? Et par-là de son incapacité à fonder les conditions théoriques préalables à une émancipation humaine véritable?

\section{NOTES}

1. Danilo Martuccelli, Sociologies de la modernité : l'itinéraire du XXe siècle, Gallimard, Paris, 1999, p. 551.

2. Selon la formule de Merleau-Ponty pour décrire ces marxismes hétérodoxes plutôt centrés sur l'analyse philosophique de l'œuvre du jeune Marx. Voir : Maurice Merleau-Ponty, Les aventures de la dialectique, Gallimard, Paris, 2000.

3. Axel Honneth, La réification : petit traité de théorie critique, Gallimard, Paris, 2007.

4. György Lukács, Histoire et conscience de classe : essais de dialectique marxiste, Éditions de Minuit, Paris, 1976.

5. Michael Löwy, Marxisme et romantisme révolutionnaire : essais sur Lukács et Rosa Luxemburg, Le Sycomore, Paris, 1979.

6. Kostas Alexos, "Préface à 'Histoire et conscience de classe : essais de dialectique marxiste' ", Lukács, op. cit.

7. Youssef Ishaghpour, « Avant-propos à Lukács et Heidegger : pour une nouvelle philosophie. Fragments posthumes", Youssef Ishaghpour (dir.), Lukács et Heidegger : pour une nouvelle philosophie. Fragments posthumes, Denoël/Gonthier, Paris, 1973.

8. La question est en effet disputée entre les commentateurs de Lukács de savoir si la réification est avant tout un phénomène d'ordre cognitif ou effectif. La question peut être posée en termes d'une opposition entre l'ontique et l'ontologique comme réalité du phénomène réificatoire. Pour une synthèse compète de ces deux postulats exégètes, on se référera aux travaux de Vandenberghe (voir Frédéric Vandenberghe, Une histoire critique de la sociologie allemande : aliénation et réification, La Découverte, Paris, 1997. Aussi : Frédéric Vandenberghe, « La notion de réification. Réification sociale et chosification méthodologique", L'Homme et la société, Aliénations nationales (193), 1992, pp. 81-93). 
9. Lukács, op. cit., p. 112.

10. Op. cit., p. 114.

11. Op. cit., p. 110.

12. Anselm Jappe, "Aliénation, réification et fétichisme de la marchandise ", Chanson Vincent, Alexis Cukier, Frédéric Monferrand (dir.), La réification : histoire et actualité d'un concept critique, La Dispute, Paris, 2014, p. 80.

13. Au sens de la perte dans les processus de travail de tout ce qui fait l'individu concret, et le différencie d'autrui. Cette différentiation n'est absolument pas pertinente dans la sphère du travail capitaliste. Les différences intrinsèques (identitaires) de l'individu n'étant en rien fonctionnel, voire étant potentiellement dysfonctionnelles au sein de processus de production de plus en plus standardisés, il importe de les supprimer du champ de vision de la relation capitaliste moderne.

14. Lukács, op. cit., p. 117.

15. Op. cit., p. 129.

16. Ibidem

17. Karl Marx, Friedrich Engels, "Le Manifeste communiste ", Karl Marx, Euvres, Gallimard, Paris, 1977, p. 168.

18. Lukács, op. cit., p. 117.

19. Op. cit., p. 119.

20. Herbert Marcuse, Pour une théorie critique de la société. Contre la force répressive, Denoël/ Gonthier, Paris, 1971, p. 136.

21. György Lukács, Entretiens avec Georg Lukács, Libraire François Maspero, Paris, 1969.

22. Stieg Dagerman, Notre besoin de consolation est impossible à rassasier, Actes Sud, Arles, 1989, p. 20.

23. Henri Lefebvre, «Introduction à Marx, 1818-1883. », Henri Lefebvre (dir.), Marx, 1818-1883 / Introduction et choix par Henri Lefebvre, Éditions des Trois Collines, Genève, 1947, p. 109.

24. Voir : Axel Honneth, La lutte pour la reconnaissance, Gallimard, Paris, 2000.

25. Axel Honneth, La réification : petit traité de théorie critique, Gallimard, Paris, 2007, p. 18.

26. Axel Honneth, «Réification, connaissance, reconnaissance : quelques malentendus », Esprit, Juillet (7), 2008, p. 92.

27. Axel Honneth, La réification : petit traité de théorie critique, Gallimard, Paris, 2007, p. 28.

28. Emmanuel Renault, L'expérience de l'injustice. Reconnaissance et clinique de l'injustice, La Découverte, Paris, 2004, p. 54.

29. Voir : Stéphane Haber, «Renouveau de la philosophie sociale ?", Esprit, Mars-avril (3), 2012. Voir aussi : Emmanuel Renault, L'expérience de l'injustice. Essai sur la théorie de la reconnaissance, La Découverte, Paris, 2017.

30. Axel Honneth, La lutte pour la reconnaissance, Gallimard, Paris, 2000, p. 147.

31. Axel Honneth, La société du mépris. Vers une nouvelle Théorie critique, La Découverte / Poche, Paris, 2014, p. 189.

32. Honneth, op. cit., p. 220.

33. Axel Honneth, Olivier Voirol, «La théorie critique de l'École de Francfort et la théorie de la reconnaissance", Axel Honneth, La société du mépris. Vers une nouvelle Théorie critique, La Découverte / Poche, Paris, 2014, p. 171.

34. Honneth, Voirol, op. cit., p. 173.

35. Axel Honneth, La réification : petit traité de théorie critique, Gallimard, Paris, 2007, p. 52.

36. Honneth, op. cit., p. 81.

37. La réification comme oubli de la reconnaissance permet conceptuellement de ne pas sombrer dans l'impasse lukácsienne, et francfortoise, de la société totalement réifiée comme ayant perdu tout contact avec la praxis authentique. La question n'est alors plus celle 
d'une pratique inauthentique qui aurait remplacé la pratique authentique : la pratique est restée, nécessairement, mais est comprise de manière non correcte, ce qui en transforme le sens, et ensuite la transforme. De là provient la nécessité théorique de fonder le diagnostic sur une analyse phénoménologique de la réification. Honneth entend dégager ce qu'est la pratique non-réifiée, victime de l'oubli, hors des développements spéculatifs sur la (prise de) conscience prolétarienne. Pour Lukács, même en admettant la réification comme absence d'engagement empathique de l'homme à l'égard de son monde, il n'est en aucun cas question d'oubli ou d'aveuglement mais plutôt de la perte du sens de la totalité, social et historique, au profit d'une suite de factualités séparées.

38. Notons par honnêteté intellectuelle qu'Honneth n'abandonne pas totalement et irrémédiablement la causalisation marchande capitaliste. De temps en temps en effet, le philosophe introduit quelque chose de l'ordre de la causalité marchande, comme ici : «[d]ans la sphère toujours en expansion de l'échange marchand, les sujets sont contraints de se comporter par rapport à la vie sociale, en observateurs distanciés plutôt qu'en participants actifs, parce que tous les calculs qu'ils font au cours de ces actions et à propos de ce qu'ils pourraient obtenir les uns des autres exigent une position purement rationnelle et aussi exempte d'émotions que possible » (dans : La réification : petit traité de théorie critique, op. cit., p. 26.). On a ici également une intuition de l'étiologie capitaliste de la réification : « [1]e rapport social y est pensé dans son ensemble selon le modèle de la transaction économique entre individus économiques indépendants (unabhängigen Wirtschaftsbürgern), de sorte qu'il ne reste plus d'espace pour des sphères de la société qui s'obstinent à fonctionner selon d'autres principes ou orientations que ceux de la rentabilité économique. De là vient l'importance considérablement accrue des nouveaux modèles de management, des budgétisations et des calculs économiques, qui semblent à l'heure actuelle pénétrer la vie de la société par tous ses pores " (dans: "Réification, connaissance, reconnaissance : quelques malentendus », art. cit., p. 95). Il n'en reste pas moins que celle-ci est relativement diluée au sein d'un ensemble causal large et d'origine plutôt culturelle.

39. Axel Honneth, La réification : petit traité de théorie critique, Gallimard, Paris, 2007, p. 114.

40. Honneth, op. cit., p. 115.

41. Op. cit., p. 116.

42. David Owen, «Reification, ideology and power: expression and agency in Honneth's theory of recognition ", Journal of Power, 3(1), 2010, p. 103.

43. Christian Lazzeri, «Réification et reconnaissance. Une discussion avec Axel Honneth. », Revue du MAUSS, 38(2), 2010, p. 281.

44. Axel Honneth, La réification : petit traité de théorie critique, Gallimard, Paris, 2007, p. 115.

45. Il faut rendre à Honneth qu'il concédera lui-même postérieurement qu'il s'agit d'un mauvais exemple.

46. Axel Honneth, «Réification, connaissance, reconnaissance: quelques malentendus », Esprit, Juillet (7), 2008, p. 106.

47. Lazzeri, op. cit., p. 261.

48. Marco Angella, «Les limites du concept de réification chez Honneth» Vincent Chanson, Alexis Cukier, Frédéric Monferrand (dir.), op. cit., p. 335.

49. Timo Jütten, «What is Reification? A Critique of Axel Honneth», Inquiry, 53(3), 2010, pp. 235256.

50. Vincent Charbonnier, «La réification chez Lukács (la madeleine et les cendres) », Chanson Vincent, Alexis Cukier, Frédéric Monferrand (dir.), op. cit., pp. 43-65.

51. Alastair Morgan, «The « living entity »: Reification and forgetting », European Journal of Social Theory, 17(4), 2014, pp. 377-388. 
52. György Lukács, Histoire et conscience de classe : essais de dialectique marxiste, Éditions de Minuit, Paris, 1976, p. 123.

53. Ibidem

54. Lukács, op. cit., p. 135.

INDEX

Mots-clés : critique, réification, marxisme, théorie de la reconnaissance, théorie sociale

\section{AUTEUR}

\section{ANTOINE PRINTZ}

Membre du CriDIS, Université catholique de Louvain 